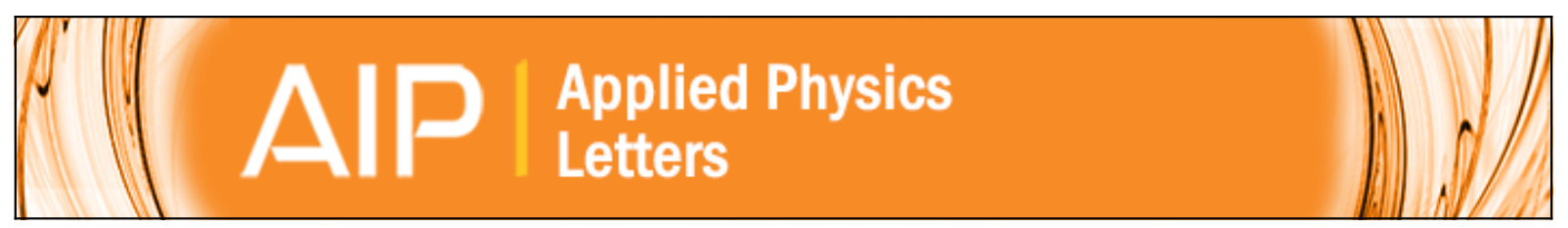

\title{
Ductility and work hardening in nano-sized metallic glasses
}

D. Z. Chen, X. W. Gu, Q. An, W. A. Goddard III, and J. R. Greer

Citation: Applied Physics Letters 106, 061903 (2015); doi: 10.1063/1.4907773

View online: http://dx.doi.org/10.1063/1.4907773

View Table of Contents: http://scitation.aip.org/content/aip/journal/apl/106/6?ver=pdfcov

Published by the AIP Publishing

\section{Articles you may be interested in}

Fe-based bulk metallic glasses: Brittle or ductile?

Appl. Phys. Lett. 105, 161901 (2014); 10.1063/1.4899124

Plastic stability of metallic glass composites under tension

Appl. Phys. Lett. 103, 151910 (2013); 10.1063/1.4824879

Pronounced ductility in CuZrAl ternary bulk metallic glass composites with optimized microstructure through melt adjustment

AlP Advances 2, 032176 (2012); 10.1063/1.4754853

Work hardening ability of ductile Ti $45 \mathrm{Cu} 40 \mathrm{Ni} 7.5 \mathrm{Zr} 5 \mathrm{Sn} 2.5$ and $\mathrm{Cu} 47.5 \mathrm{Zr} 47.5$ Al 5 bulk metallic glasses Appl. Phys. Lett. 89, 071908 (2006); 10.1063/1.2337534

Ductility of bulk nanocrystalline composites and metallic glasses at room temperature Appl. Phys. Lett. 77, 46 (2000); 10.1063/1.126872

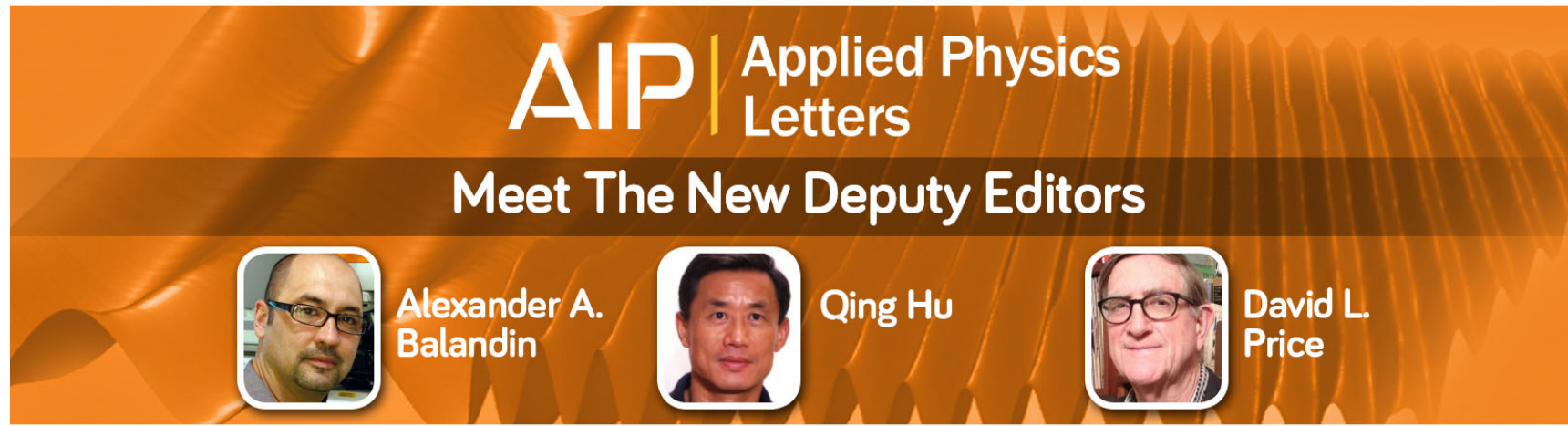




\title{
Ductility and work hardening in nano-sized metallic glasses
}

\author{
D. Z. Chen, ${ }^{1, a)}$ X. W. Gu, ${ }^{2}$ Q. An, ${ }^{3}$ W. A. Goddard III, ${ }^{3}$ and J. R. Greer ${ }^{1,4}$ \\ ${ }^{1}$ Division of Engineering and Applied Sciences, California Institute of Technology, Pasadena. \\ California 91125, USA \\ ${ }^{2}$ Department of Chemistry and Chemical Engineering, California Institute of Technology, Pasadena, \\ California 91125, USA \\ ${ }^{3}$ Materials and Process Simulation Center, California Institute of Technology, Pasadena, \\ California 91125, USA \\ ${ }^{4}$ The Kavli Nanoscience Institute, California Institute of Technology, Pasadena, California 91125, USA
}

(Received 5 December 2014; accepted 28 January 2015; published online 9 February 2015)

In-situ nano-tensile experiments on $70 \mathrm{~nm}$-diameter free-standing electroplated NiP metallic glass nanostructures reveal tensile true strains of $\sim 18 \%$, an amount comparable to compositionally identical $100 \mathrm{~nm}$-diameter focused ion beam samples and $\sim 3$ times greater than $100 \mathrm{~nm}$-diameter electroplated samples. Simultaneous in-situ observations and stress-strain data during post-elastic deformation reveal necking and work hardening, features uncharacteristic for metallic glasses. The evolution of free volume within molecular dynamics-simulated samples suggests a free surfacemediated relaxation mechanism in nano-sized metallic glasses. () 2015 AIP Publishing LLC.

[http://dx.doi.org/10.1063/1.4907773]

In 1960, Klement et al. revealed the possibility of a stable amorphous phase for a binary metallic alloy, obtainable via extremely rapid cooling. ${ }^{1}$ Since then, such amorphous metal alloys, or metallic glasses (MG), with two or more components have been fabricated and tested for their mechanical properties, and represent materials with high strength, high elastic limit, and superior fatigue resistance. ${ }^{2}$ However, due to their random packing and a lack of known plasticity carriers, such as dislocations in their crystalline counterparts, failure in room-temperature monolithic bulk metallic glasses under tension is typically catastrophic at the elastic limit, ${ }^{3}$ and significant research has been directed at toughening and at inducing ductility into these very strong, yet brittle materials.

Some successful attempts to toughen MG have involved the use of heterostructures and composites to impede shear band propagation or to promote the formation of multiple diffuse, non-catastrophic, and shear bands. ${ }^{4-7}$ These methods were shown to be effective in delaying failure, but the formulation of intrinsic physical mechanisms responsible for sustainable plasticity under tensile loading has been sparse. Recent in-situ observations of a brittle-to-ductile transition emergent in nanometer-sized metallic glasses under tension suggest a mechanism for plasticity not involving shear bands. ${ }^{8-11}$ In one such work, Jang et al. demonstrated that $\mathrm{Zr}_{35} \mathrm{Ti}_{30} \mathrm{Co}_{6} \mathrm{Be}_{29}$ metallic glass nano-cylinders underwent a transition from catastrophic shear banding in samples with diameters greater than $100 \mathrm{~nm}$ to a ductile necking-to-shearbanding deformation prior to failure in thinner samples. Stress-strain data in these samples show distinct work hardening to $\sim 25 \%$ true strain and permanent plastic deformation upon unloading past the elastic limit. ${ }^{9}$ Similar plasticity and necking have been reported for electroplated (EP) NiP MGs with diameters of $100 \mathrm{~nm}$ and below that were fabricated with

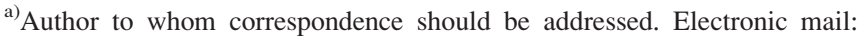
dzchen@caltech.edu
}

and without the use of focused ion beam (FIB),${ }^{10}$ as well as for melt-spinned CuZr MGs with diameters 70-120 nm fabricated with FIB. ${ }^{11}$ Necking and strain hardening are typical for crystalline metals and metal alloys, whose plastic flow is enabled by the motion and interactions of dislocations but are anomalous for amorphous metals. These findings leave us with two important open questions: (1) What is responsible for the plastic flow and (2) why does strain hardening occur? It has been speculated that size-induced brittle-to-ductile transition may be caused by a surface modulated mechanism that governs extendibility, an idea supported by the additional enhancement in ductility in ion-irradiated samples. ${ }^{9,10,12}$ Such a mechanism would have consequences for both the sample size as well as sample surface energy state. To explore the physical origins of emergent plasticity and hardening in nano-sized metallic glasses, we performed insitu nano-tensile experiments on $\sim 70 \mathrm{~nm}$-diameter electroplated NiP MG samples, whose surface area-to-volume ratio is $\sim 0.06$, representing a $50 \%$ enhancement in the relative role of the sample surface compared to 0.04 for $\sim 100 \mathrm{~nm}$ samples.

To fabricate the $70 \mathrm{~nm}$-diameter $\mathrm{NiP}$ samples, we employed similar fabrication parameters as in Ref. 10 with a reduced pore size in the templates. This enabled the fabrication of chemically identical but smaller electroplated NiP MG samples (see Table I). Figure 1 shows an array of electroplated pillars (Fig. 1(a)) as well as a schematic of the fabrication process (Fig. 1(b)). Repeatability of the electroplating process suggests that the $70 \mathrm{~nm}$-diameter pillars studied here

TABLE I. Geometry and composition of samples.

\begin{tabular}{lcrr}
\hline \hline Sample & Composition (wt. \%) & Diameter (nm) & Length (nm) \\
\hline $100 \mathrm{~nm}$ FIB & P-14.0 Ni-86.0 & $93.4 \pm 7.77$ & $598.2 \pm 21.97$ \\
$100 \mathrm{~nm}$ EP & P-14.9 Ni-85.1 & $104.8 \pm 6.46$ & $633.0 \pm 76.57$ \\
$70 \mathrm{~nm}$ EP & P-14.9 Ni-85.1 & $68.2 \pm 2.98$ & $433.9 \pm 10.19$ \\
\hline \hline
\end{tabular}




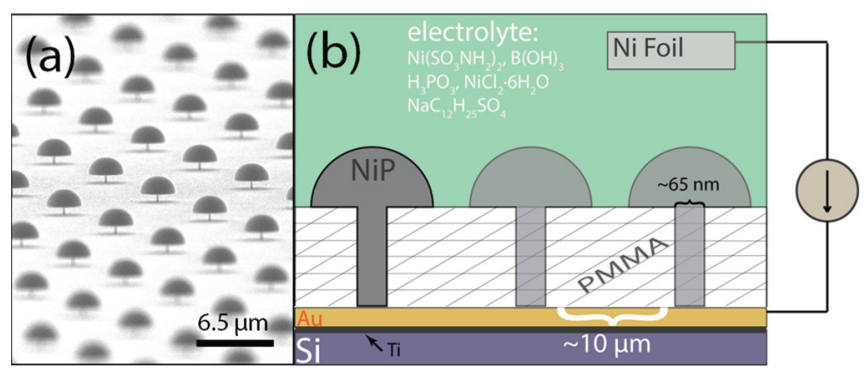

FIG. 1. (a) Electroplated MG samples. (b) Schematic of the template electroplating procedure.

have nominally the same chemical composition and amorphous microstructure as the $100 \mathrm{~nm}$-diameter samples. ${ }^{10}$

Uniaxial tensile experiments were performed in a custom-made in situ scanning electron microscope (SEM) with a nanomechanical module, InSEM ${ }^{\mathrm{TM}}$ (Nanomechanics, Inc.), at a constant nominal displacement rate (0.4-0.6 $\mathrm{nms}^{-1}$ ), resulting in a global strain rate of $\sim 0.001 \mathrm{~s}^{-1}$. The ultimate tensile strengths were comparable across all samples: $1.92 \pm 0.14 \mathrm{GPa}$ for $70 \mathrm{~nm}-\mathrm{EP}$ studied in this work; $1.87 \pm 0.38 \mathrm{GPa}$ for $100 \mathrm{~nm}-\mathrm{EP}$ and $1.91 \pm 0.36 \mathrm{GPa}$ for $100 \mathrm{~nm}$ FIB-carved samples from Ref. 10. Plastic strain, defined as $\varepsilon_{\mathrm{p}}=\varepsilon_{\text {total }}-\varepsilon_{\text {elastic }}$, was nearly the same for the $70 \mathrm{~nm}-\mathrm{EP}$ and $100 \mathrm{~nm}-\mathrm{FIB}$ samples at $2.0 \% \pm 0.5 \%$ and $2.0 \% \pm 1.0 \%$, respectively. These values are $\sim 2.5$ times higher than the plastic strain for $100 \mathrm{~nm}-\mathrm{EP}$ samples, $\sim 0.8 \% \pm 0.4 \%$. Elastic moduli were consistent across all samples: $71.4 \pm 32.9 \mathrm{GPa}$ for $70 \mathrm{~nm} \mathrm{EP}, 63.6 \pm 13.4 \mathrm{GPa}$ for $100 \mathrm{~nm}-\mathrm{FIB}$, and $60.3 \pm 17.7 \mathrm{GPa}$ for $100 \mathrm{~nm}-\mathrm{EP}$. Contrary to the catastrophic failure via shear banding at the elastic limit often seen in large MG samples under tension, the $70 \mathrm{~nm}$ diameter samples studied in this work show nonlinear plasticity of $\sim 2 \%$ engineering strain ( $\sim 14 \%$ true strain) along with necking and work hardening, characteristics typically associated with ductile crystalline metals and alloys. Other observations of necking in nano-sized metallic glasses have been reported, but are limited, occurring in $100 \mathrm{~nm}$ FIBmilled Zr-based MG structures (set of three), ${ }^{8}$ isolated FIBmilled $100 \mathrm{~nm}$ Zr-based pillars, ${ }^{10}$ isolated FIB-milled 70-120 nm CuZr samples, ${ }^{11}$ and isolated FIB-exposed Ptbased MG wires. ${ }^{12}$ In distinction, our samples are simultaneously freestanding, smaller, and FIB-free.

Figure 2 shows SEM images of a typical as-plated sample (Fig. 2(a)) and a time-lapsed progression from the in-situ tension test (Figs. 2(b)-2(f)). The corresponding engineering and true stress-strain data are provided for the representative sample (Figs. 2(g) and 2(h)). True stresses and strains were obtained from measuring the sample diameter in the neck region by performing image analysis on the in-situ SEM video snapshots (Fig. 3(b)). Average values for engineering ultimate tensile strength and plastic strain for the seven tested samples, on which all statistics are based, are provided in Figure 3, along with those for the $100 \mathrm{~nm}$-diameter samples from Ref. 10 for comparison.

One mode of deformation in MGs is the spontaneous collective rearrangements of $\sim 10-20$-atom clusters, ${ }^{13-18}$ commonly referred to as shear transformation zones (STZs). ${ }^{19,20}$ Room-temperature tensile plasticity in metallic glasses is typically nonexistent because in these conditions the STZs strain-soften and coalesce quickly to form shear bands that lead to sample failure. Homogeneous deformation in monolithic bulk metallic glasses (BMGs) normally only occurs at elevated temperatures, above or near the glass-transition, with failure marked by necking and drawing to a point instead of shear banding. ${ }^{3,21-23}$ The 70 nm-diameter EP
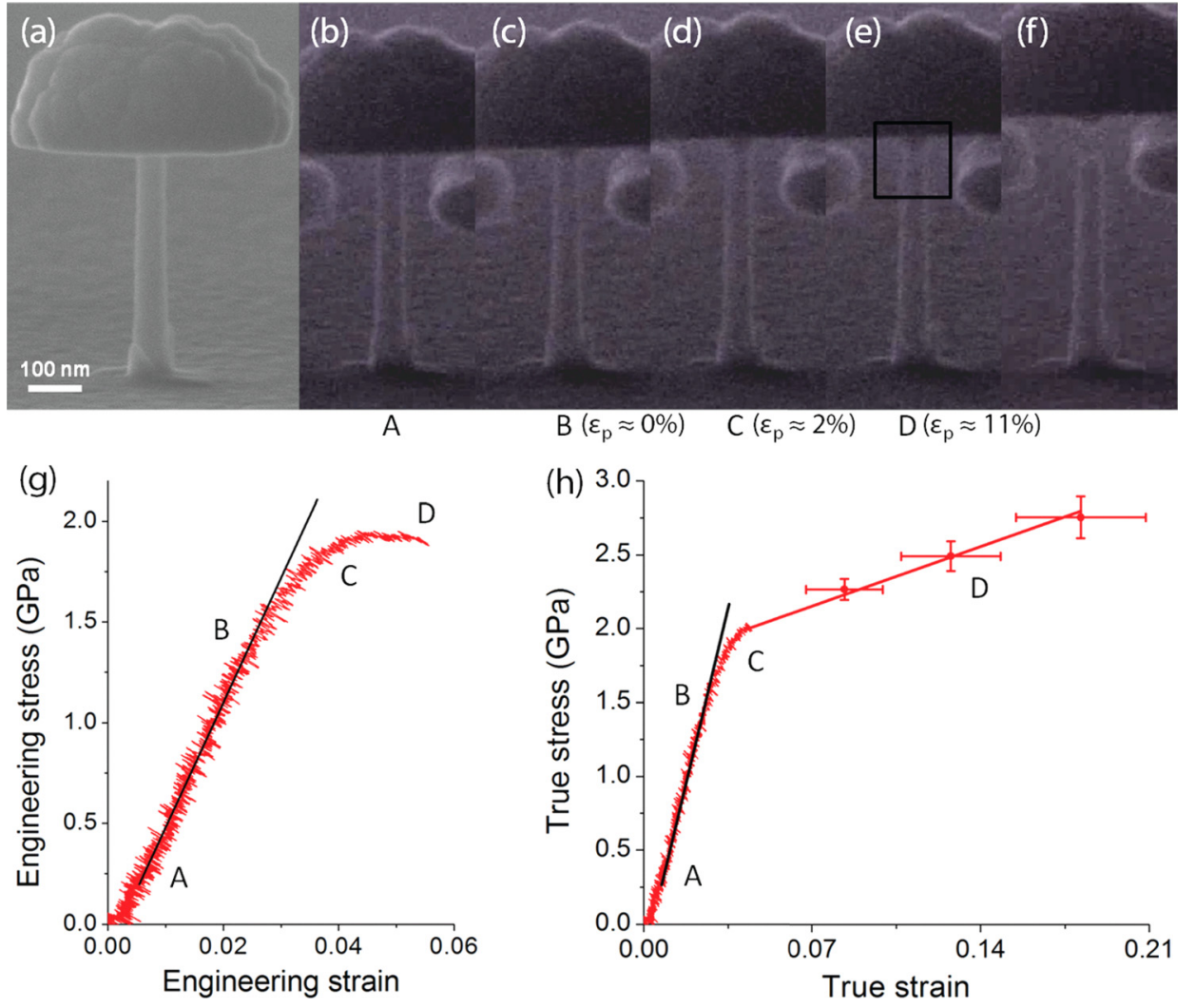

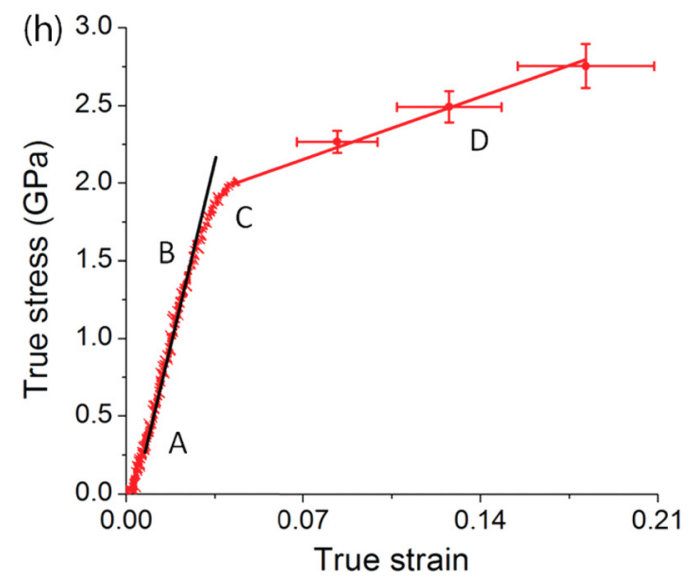

FIG. 2. (a)-(f) Corresponding in-situ SEM micrograph snapshots of the deformation-contrast adjusted. Necking can be observed in the boxed region of panel (e). (g) and (h) Engineering and true stress strain curves with corresponding SEM images at A: initial loading, B: elastic limit, C: plasticity, and D: necking prior to failure. Error bars represent measurement error of diameter in necked region. 
(a)

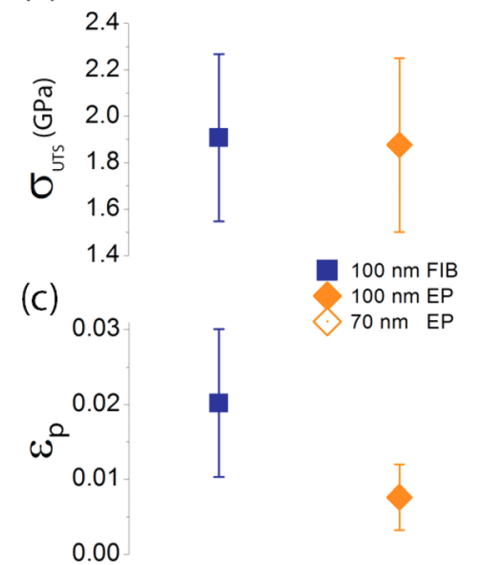

(b)

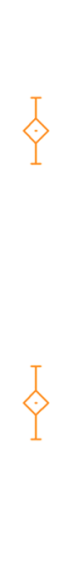

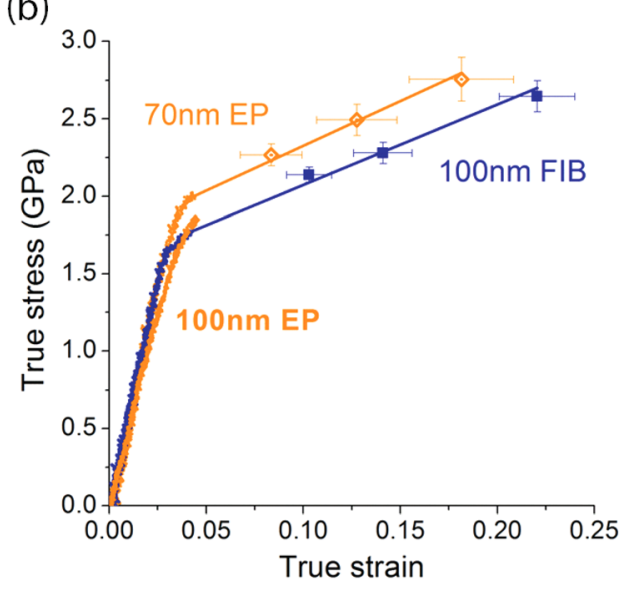

FIG. 3. (a) Ultimate tensile strengths for all samples. (b) True stress strain comparison. (c) Engineering plastic strains. samples studied in this work, as well as the $100 \mathrm{~nm}$-diameter FIB and EP samples, ${ }^{10}$ show significant post-elastic deformability at room temperature, yet still fail via propagation of a single shear band. This demonstrates that nano-size metallic glasses (nano-MGs) with diameters of 70-100 $\mathrm{nm}$ behave in a way that is simultaneously distinctive to room-temperature failure and to near $\mathrm{T}_{\mathrm{g}}$-temperature plastic flow in tensed monolithic bulk MGs. What sets them apart is the emergence of work hardening, which suggests that an internal hardening mechanism exists in nano-MGs.

The competing energy of crack-like shear band propagation and homogeneous plastic flow has been used to explain the suppression of shear band propagation in nanoMGs., 92,24 This is helpful in understanding the general deformation phenomena but lacks information about the underlying physical processes that drive plasticity and work hardening. To gain insight into these physical processes, we carried out Molecular Dynamics (MD) simulations on $30 \mathrm{~nm}$-diameter $\mathrm{Ni}_{80} \mathrm{Al}_{20}$ metallic glass cylinders using an embedded atom method (EAM) potential ${ }^{25}$ in LAMMPS. $^{26}$ Cylinders were cut from bulk MG made by simulated quenching; periodic boundaries were used in the axial direction, with the cylinder surfaces free. The strain rate was $10^{8} \mathrm{~s}^{-1}$, a value that represents a compromise between qualitative accuracy and feasible computational time. ${ }^{27} \mathrm{~A}$ Ni-Al EAM potential was chosen to be consistent with our previous work in Ref. 10. Ni-Al and Ni-P glasses possess different bonding types, which may give rise to differences in failure modes. For example, in metal-metalloid compounds (e.g., Ni-P) cavitation-induced brittle failure has been observed depending on the degree of stress triaxiality. ${ }^{28,29}$ Despite these differences in bonding characteristics, the free volume dynamics of Ni-P and Ni-Al are likely similar because both systems are amorphous. Earlier we mentioned that sample surface energy state plays an important role in the brittleto-ductile transition, ${ }^{10,12}$ so we tracked the evolution of free volume distribution across two different sample types: (1) as-cast (Figs. 4(a) and 4(c)) and (2) irradiated (Figs. 4(b) and $4(d)$ ), as a function of tensile strain. Free volume was chosen as an effective parameter for the extent of relaxation and not intended to invoke free volume theory, ${ }^{22,30}$ which may have pitfalls when used for non-Van der Waals systems. ${ }^{31,32}$ In our metallic system, "free volume" is more appropriately a measure of the local densities or inferred atomic level stresses. ${ }^{33,34}$ We binned the pillars into $1 \AA$-thick concentric hollow cylinders, and using a simple Voronoi tessellation (Fig. 4(e)), estimated the free volume, or local density, distribution by an excess Voronoi volume, defined as the normalized average binned Voronoi volume at each strain $(0 \%, 1 \%$, $3 \%, 5 \%$, and $7 \%$ ) minus the mean Voronoi volume over the whole system at the initial configuration ( $0 \%$ strain). The irradiated sample initially had $\sim 2 \%-3 \%$ higher free volume, mostly localized within $0.6-0.9 \mathrm{~d} / \mathrm{d}_{\max }$ away from the center, compared to the as-cast sample because of the collision cascades caused by the irradiation process. The computations reveal that during initial elastic loading ( $0 \%$ to $\sim 5 \%$ strain), the free volume in both samples increased uniformly and isotropically in the core, $d / d_{\max }<0.95$ (Figs. 4(c) and 4(d)). This can be attributed to bond length dilation associated with elastic deformation. Past $\sim 5 \%$ strain, the free volume evolved differently in the core region, $\mathrm{d} / \mathrm{d}_{\max }<0.95$, compared with the near-surface region, $d / d_{\max }>0.95$ (Figs. 4(a) and $4(\mathrm{~b})$ ). The core region attained saturation in free volume despite the local atomic strains showing significant activity, ${ }^{10}$ meaning the atoms had rearranged but no free volume was accumulated overall. In contrast, this saturation does not occur in the near-surface region, $\mathrm{d} / \mathrm{d}_{\max }>0.95$, of the cylindrical samples nor does it occur in a bulk sample (Fig. 4(f)) with periodic boundaries. Conservation of total volume during plastic deformation is expected in metals where crystallographic slip via shear processes gives rise to plasticity. It does not necessarily hold for metallic glasses, whose common mechanisms of atomic-scale deformation, STZ-type and diffusive-jump-type, are both dilatational. ${ }^{3}$ A semipermanent local increase in volume occurs in the course of either mechanism in addition to a transient dilatation that is necessary to overcome saddle points in the energy landscape. ${ }^{3}$ In our results on bulk NiAl metallic glass with periodic boundaries in all directions, the Voronoi volume continues to increase during plasticity, after loading past $5 \%$ strain (Fig. 4(f)), albeit at a different rate than during elastic loading. Following this line of reasoning, maintaining a constant volume distribution in the pillar cores requires that the near-surface regions absorb the local dilatational processes, which leads to a mechanism where the outer region adjacent to the free surface may serve as a free volume "sink."

We propose the following mechanism for plasticity: the core atoms undergo local dilatations, which subsequently 
(a)

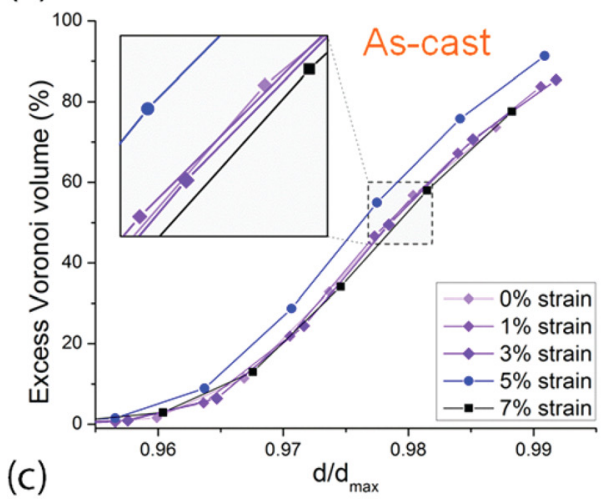

(c)

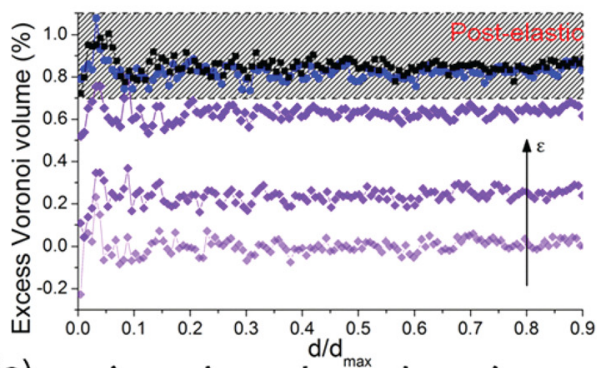

(e)

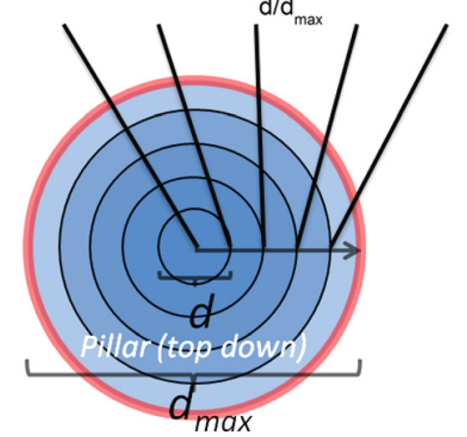

(b)
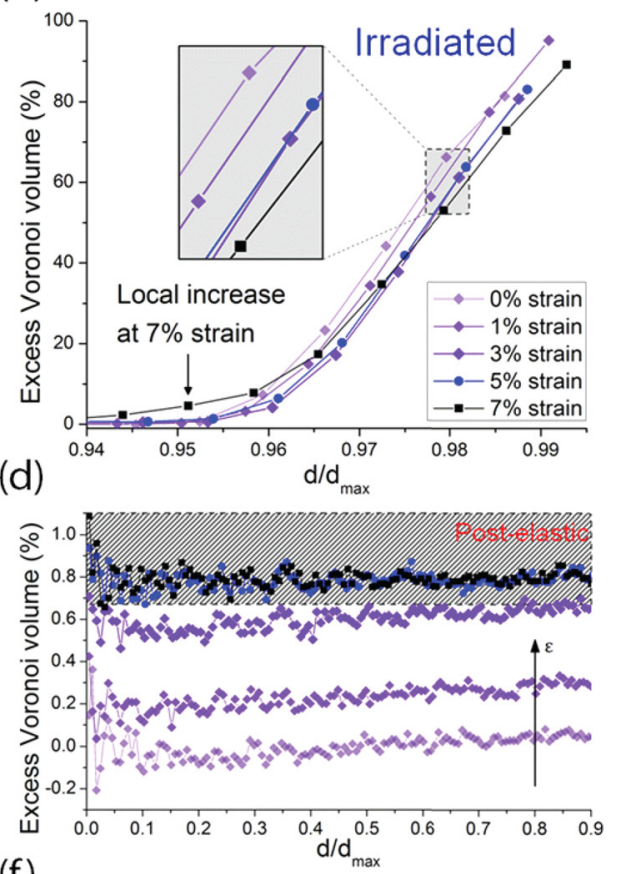

(f)

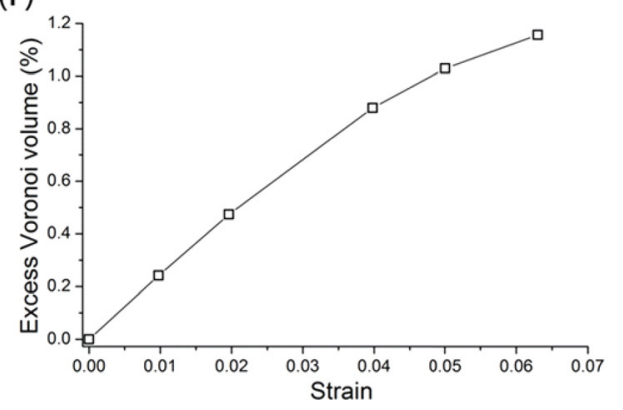

FIG. 4. Excess Voronoi volume in the ((a) and (b)) near surface region, outer $5 \%-6 \%$ of pillar, and the ((c) and (d)) core region for the as-cast system ((a) and (c)) and the irradiated system ((b) and (d)). The grated region indicates when plasticity occurs. Each point in plots (a)-(d) represents values averaged over a $1 \AA$ Åthick hollow cylindrical bin. (e) A coarse-grained top-down schematic of the binning procedure. The core region, blue, is plotted in (c) and (d), while the near-surface region, red outer ring, is plotted in (a) and (b). (f) Excess Voronoi volume versus strain for a bulk NiAl system. rearrange and annihilate near the free surface. This process, involving a relaxation through the free surface, is consistent with the observed $\sim 10 \%$ increase in the excess volume between $3 \%$ and $5 \%$ strain followed by a $\sim 10 \%$ decrease between $5 \%$ and $7 \%$ strain in the near-surface region of the as-cast system (Fig. 4(a)). This process may be similar to diffusional relaxation, and we can estimate that it occurs at a faster rate than shear localization, using free volume dynamics theory. ${ }^{22,27}$ The ability of the atomic clusters in the vicinity of the free surface to absorb excess free volume may derive from their relatively unconstrained access to lower local free volume configurations. We postulate that the depletion in the overall free volume within the core of the pillar caused by this relaxation delays shear localization and temporarily shifts the deformation to a quasi-homogeneous mode. Following this phenomenological description, the observed size effects of ductility and hardening may emanate from the higher surface area-to-volume ratio in smallerdiameter cylinders, which allows the entire sample to undergo a more homogenous-like deformation before significant shear localization occurs.

Enhanced ductility has also been reported for nano-sized samples whose surfaces have been bombarded with ions (e.g., during FIB-milling), ${ }^{9,10,12}$ increasing the energy of the free surface by generating excess free volume near the surface and inducing a relatively homogeneous distribution of saddle-point configurations. In our simulations, irradiated samples show a local decrease in the free volume during elastic loading (Fig. 4(b)) and in the relaxation of the saddlepoint configurations near the surface. Between 3\% and 5\% strain, the free volume in the near-surface region no longer decreases, which suggests a competition between the free volume generation due to dilatation and the loss of free volume at the near-surface sink. More revealing is the process between $5 \%$ and $7 \%$ plastic strain, where a local volume increase of $\sim 5 \%$ is seen farther away from the free surface than in the as-cast sample, $\mathrm{d} / \mathrm{d}_{\max } \sim 0.95$ (indicated by black arrow, Fig. 4(b)), and relaxation again occurs closer to the free surface, $d / d_{\max }>0.98$. These observations suggest that FIB-milling may effectively broaden the near-surface region, enabling relaxation to occur further from the free surface, reducing the diameter of the core region, and promoting homogeneous-like deformation.

Work hardening in the nano-sized MGs may stem from relaxation through atomic arrangements in the outer surface region leading to a deficiency of free volume in the core of the nano-sized cylinder and suppressing the coalescence of shear-softened regions into a catastrophic shear band. This occurs after the rate of relaxation through the surface becomes comparable with the rate of local dilatation events within the sample, which results in a necked region with quasihomogeneous flow and a state of free volume "starvation," 
somewhat analogous to dislocation starvation in single crystalline nanopillars. ${ }^{35}$ Hardening may be the natural result of the densification and relaxation of local dilatations, similar to what is seen in notched bulk MGs under induced multiaxial stress states. ${ }^{28,36}$ The complexity of metallic glasses prevents a more rigorous explanation of the relaxation mechanism, as the deformation modes in MGs are still not fully understood, but this phenomenological description attempts to capture all the salient experimental observations.

In addition to its complex microstructure, another major challenge to studying amorphous metals is the apparent disconnect in deformation mechanisms between BMGs and nano-MGs. When tensed at room temperature, the only plasticity mechanism present in macro-sized monolithic samples is failure via one or more catastrophic shear bands. ${ }^{3}$ At the nanoscale, tensile failure exhibits a size effect, which is manifested by a transition from brittle-like shear band propagation in larger samples (i.e., greater than $\sim 100 \mathrm{~nm}$ ) to a more ductile mode, in which shear banding is suppressed and necking/work hardening is observed. ${ }^{8-10}$ These differences likely stem from the rate dependence of an amorphous system on the structural dynamics of being driven from its metastable state. In bulk samples, at room temperature, the system cannot relax appreciably in response to strain energy because dissipation mechanisms are kinetically limited, whereas in a nano-sized sample this may occur through the free surface. The implication of such a mechanism is that at room temperatures nano-MGs exhibit mechanical properties foreign to monolithic BMGs, i.e., ductility and work hardening, and that these properties can be obtained by simply tuning the surface area to volume ratio. The idea that plasticity in metallic glasses is dependent on structural dynamics is also supported by various experiments showing its strain-rate and temperature dependence. Slower strain rates and higher temperatures, marked by viscous homogeneous flow, typically lead to more ductile metallic glasses. ${ }^{3,21-23}$

Plasticity in BMGs can be achieved through impeding shear band propagation, ${ }^{6,7}$ and plasticity in nano MGs emerges due to size reduction. ${ }^{9,10}$ Both of these separate yet related mechanisms can be utilized in conjunction by using an architectural approach to material design. For example, nano-sized MG heterostructures, or nanopores, may be able to exploit both bulk and nano-scale plasticity mechanisms to suppress shear banding as well as impede the propagation of existing shear bands, and architected MG nanolattices can utilize both structural and material effects to fill untouched regions of the material design space. ${ }^{37-39}$ A mastery of engineered hierarchy in material microstructures might one day allow us to make ductile metallic glasses that both deform and harden like steels and possess superior strength and stiffness.

The authors acknowledge summer students Boyu Fan and Timothy Tsang for their help with electroplating. The authors gratefully acknowledge the financial support of the U.S. Department of Energy, Office of the Basic Energy Sciences, and NASA's Space Technology Research Grant Programs through JRG's Early Career grant. The authors also acknowledge support and infrastructure provided by the Kavli Nanoscience Institute (KNI) at Caltech. All computations were carried out on the SHC computers (Caltech Center for Advanced Computing Research) provided by the Department of Energy National Nuclear Security Administration PSAAP project at Caltech (DE-FC52-08NA28613) and by the NSF DMR-0520565 CSEM computer cluster. This material is based upon work supported by the National Science Foundation Graduate Research Fellowship under Grant No. DGE-1144469. Any opinion, findings, and conclusions or recommendations expressed in the material are those of the authors and do not necessarily reflect the views of the National Science Foundation.

${ }^{1}$ W. Klement, R. H. Willens, and P. Duwez, Nature 187(4740), 869-870 (1960).

${ }^{2}$ M. F. Ashby and A. L. Greer, Scr. Mater. 54(3), 321-326 (2006).

${ }^{3}$ C. A. Schuh, T. C. Hufnagel, and U. Ramamurty, Acta Mater. 55(12), 4067-4109 (2007).

${ }^{4}$ H. Ma, J. Xu, and E. Ma, Appl. Phys. Lett. 83(14), 2793-2795 (2003).

${ }^{5}$ Y. M. Wang, J. Li, A. V. Hamza, and T. W. Barbee, Proc. Natl. Acad. Sci. U.S.A. 104(27), 11155-11160 (2007).

${ }^{6}$ D. C. Hofmann, J. Y. Suh, A. Wiest, G. Duan, M. L. Lind, M. D. Demetriou, and W. L. Johnson, Nature 451(7182), 1085-U1083 (2008).

${ }^{7}$ B. Sarac and J. Schroers, Nat. Commun. 4, 2158 (2013).

${ }^{8}$ H. Guo, P. F. Yan, Y. B. Wang, J. Tan, Z. F. Zhang, M. L. Sui, and E. Ma, Nat. Mater. 6(10), 735-739 (2007).

${ }^{9}$ D. Jang and J. R. Greer, Nat. Mater. 9(3), 215-219 (2010).

${ }^{10}$ D. Z. Chen, D. Jang, K. M. Guan, Q. An, W. A. Goddard, and J. R. Greer, Nano Lett. 13(9), 4462-4468 (2013).

${ }^{11}$ L. Tian, Z. W. Shan, and E. Ma, Acta Mater. 61(13), 4823-4830 (2013).

${ }^{12}$ D. J. Magagnosc, R. Ehrbar, G. Kumar, M. R. He, J. Schroers, and D. S. Gianola, Sci. Rep. 3, 1096 (2013).

${ }^{13}$ M. L. Falk, Phys. Rev. B 60(10), 7062-7070 (1999).

${ }^{14}$ A. C. Lund and C. A. Schuh, Acta Mater. 51(18), 5399-5411 (2003).

${ }^{15}$ F. Delogu, Phys. Rev. Lett. 100(25), 255901 (2008).

${ }^{16}$ D. Pan, Y. Yokoyama, T. Fujita, Y. H. Liu, S. Kohara, A. Inoue, and M. W. Chen, Appl. Phys. Lett. 95(14), 141909 (2009).

${ }^{17}$ J. D. Ju, D. Jang, A. Nwankpa, and M. Atzmon, J. Appl. Phys. 109(5), 053522 (2011).

${ }^{18}$ I. C. Choi, Y. Zhao, B. G. Yoo, Y. J. Kim, J. Y. Suh, U. Ramamurty, and J. I. Jang, Scr. Mater. 66(11), 923-926 (2012).

${ }^{19}$ A. S. Argon, Acta Metall. 27(1), 47-58 (1979).

${ }^{20}$ M. L. Falk and J. S. Langer, Phys. Rev. E 57(6), 7192-7205 (1998).

${ }^{21}$ J. Lu, G. Ravichandran, and W. L. Johnson, Acta Mater. 51(12), 3429-3443 (2003).

${ }^{22}$ F. Spaepen, Acta Metall. 25(4), 407-415 (1977).

${ }^{23}$ J. Megusar, A. S. Argon, and N. J. Grant, Mater. Sci. Eng. 38(1), 63-72 (1979).

${ }^{24}$ C. A. Volkert, A. Donohue, and F. Spaepen, J. Appl. Phys. 103(8), 083539 (2008).

${ }^{25}$ G. P. P. Pun and Y. Mishin, Philos. Mag. 89(34-36), 3245-3267 (2009).

${ }^{26}$ S. Plimpton, J. Comput. Phys. 117(1), 1-19 (1995).

${ }^{27}$ See supplementary material at http://dx.doi.org/10.1063/1.4907773 for a discussion on strain rates in MD and rate of diffusional relaxation versus shear localization.

${ }^{28}$ X. W. Gu, M. Jafary-Zadeh, D. Z. Chen, Z. X. Wu, Y. W. Zhang, D. J. Srolovitz, and J. R. Greer, Nano Lett. 14(10), 5858-5864 (2014).

${ }^{29}$ P. Murali, T. F. Guo, Y. W. Zhang, R. Narasimhan, Y. Li, and H. J. Gao, Phys. Rev. Lett. 107(21), 215501 (2011).

${ }^{30}$ M. H. Cohen and D. Turnbull, J. Chem. Phys. 31(5), 1164-1169 (1959).

${ }^{31}$ T. Egami, JOM 62(2), 70-75 (2010).

${ }^{32}$ Y. Q. Cheng and E. Ma, Prog. Mater. Sci. 56(4), 379-473 (2011).

${ }^{33}$ T. Egami, K. Maeda, and V. Vitek, Philos. Mag. A 41(6), 883-901 (1980).

${ }^{34}$ T. Egami, Prog. Mater. Sci. 56(6), 637-653 (2011).

${ }^{35}$ J. R. Greer and W. D. Nix, Phys. Rev. B 73(24), 245410 (2006).

${ }^{36}$ Z. T. Wang, J. Pan, Y. Li, and C. A. Schuh, Phys. Rev. Lett. 111(13), 135504 (2013).

${ }^{37}$ D. C. Jang, L. R. Meza, F. Greer, and J. R. Greer, Nat. Mater. 12(10), 893-898 (2013).

${ }^{38}$ T. A. Schaedler, A. J. Jacobsen, A. Torrents, A. E. Sorensen, J. Lian, J. R. Greer, L. Valdevit, and W. B. Carter, Science 334(6058), 962-965 (2011).

${ }^{39}$ J. Rys, L. Valdevit, T. A. Schaedler, A. J. Jacobsen, W. B. Carter, and J. R. Greer, Adv. Eng. Mater. 16(7), 889-896 (2014). 